Muro de la Investigación, 2020(2): julio-diciembre

ISSN:2523-2886

Doi: https://doi.org/10.17162/rmi.v5i2.1323

\title{
Cantar para vivir: un estudio del canto oral en adolescentes
}

\author{
Singing to live: a study of oral singing in adolescents \\ Maria Andrea Cueva Espita ${ }^{1}$, Loida Córdoba González ${ }^{2}$ \\ y Rodolfo Alanía Pacovilca ${ }^{3}$ \\ ORCID iD: https//orcid.org/0000-0002-2494-6904² \\ ORCID iD: https $/ /$ orcid.org/0000-0001-9522-24713 \\ Universidad Peruana Unión, Perú ${ }^{123}$
}

Recibido: 01 de enero de 2020

Aceptado: 02 de abril de 2020

\section{Resumen}

El canto es una práctica natural del ser humano; desde la niñez hasta la adolescencia, la persona experimenta muchos cambios fisiológicos y anatómicos; no obstante, el cambio de voz provoca que muchos adolescentes no quieran continuar cantando o piensen que no pueden. Por ello, el objetivo del presente artículo es analizar las características, estrategias pedagógicas y beneficios del canto coral para los adolescentes. En esta perspectiva, se aborda el canto, luego se explica brevemente la anatomía y fisiología del mismo, adentrándose luego en la descripción de la voz y canto coral en adolescentes, didáctica del canto y los beneficios respectivos. Luego de revisar diferentes fuentes, se concluye que, a pesar de los diferentes cambios experimentados, conociendo y aplicando una técnica correcta, ayuda y desarrolla correctamente la voz, además el canto coral provee diferentes beneficios a nivel musical, académico, social, físico y de desarrollo personal.

Palabras clave: adolescencia, canto, didáctica de canto, canto coral, música

\begin{abstract}
Singing is a natural practice of the human being; From childhood to adolescence, the person experiences many physiological and anatomical changes; however, the change in voice means that many teenagers do not want to continue singing or think they cannot. For this reason, the objective of this article is to analyze the characteristics, pedagogical strategies and benefits of choral singing for adolescents. In this perspective, singing is approached, then its anatomy and physiology are briefly explained, then going into the description of the voice and choral singing in adolescents, didactics of singing and the respective benefits. After different review sources, which concludes, despite the various changes experienced, knowing and applying proper technique, help and properly develop
\end{abstract}

${ }^{1}$ Correspondencia al autor: mariacueva@upeu.edu.pe; loidacordova@upeu.edu.pe, rodolfoalania@upeu.edu.pe 
speech, including the different choral singing provides benefits musically, academic, social, physical and personal development.

Key words: adolescence, singing, didactics of singing, choral singing, music

\section{Introducción}

El canto es un medio de expresión importante para los seres humanos. Desde cantar en la ducha hasta en una carrera profesional, sin importar la calidad de sonido producido, todas las personas han vivido esta experiencia. Sciarrillo y Gianneo (1964) afirman que "la música, particularmente cantando, despierta impulsos creadores en la mente, lo cual dirige a buscar nuevos canales de la propia expresión". Los seres humanos siempre necesitan expresarse; el canto es un buen medio para hacerlo.

El canto es una actividad tan natural en la vida; en la adolescencia, la voz sufre cambios drásticos; aparece el especial cuidado, se experimentan dificultades que impiden el cuidado; además de los muchos cambios, resulta fácil perder el encanto por el canto; se adoptan malos hábitos que conducen hacia una mala ejecución, generando daño en algún órgano; finalmente, los adolescentes no quieren continuar cantando. El objetivo de este trabajo es analizar las características, estrategias pedagógicas que se deben tener en cuenta y los beneficios de la práctica coral en adolescentes.

\section{El canto y el canto coral}

Conceptos del canto

Jaraba (1989) define el canto como "una cualidad primaria del ser humano, inherente a su facultad para emitir sonidos. Todo el que puede hablar en principio puede cantar" a esto le añade entonces que, "el canto no es más que un ordenamiento lógico de las inflexiones de la voz' (p. 15).

Para Calderón (citado por Matta Alza, 2018), el canto es: "la emisión de una serie modulada de sonidos con los órganos de la voz, donde se ponen de manifiesto las características de los factores constitucionales de cada cuerpo y cada personalidad, así como la situación vital del momento" (p. 25).

Según The Oxford Handbook of Voice Perception, el canto es una "parte regular de la expresión humana. Cantar puede considerarse como un derivado -o el origen- del habla; ambos usan la misa herramienta, el órgano de la voz" (Frühholz y Belin, 2018, p. 117). 
Por su parte, Encyclopedia Britannica (2018) aporta una definición amplia al canto: "la producción de tonos musicales mediante la voz humana. En su aspecto fisico, cantar tiene una técnica bien definida que depende del uso de los pulmones, quienes actúan suministrando el aire, o fuelle; en la laringe que actúa como caña o vibrador; en las cavidades del pecho o cabeza, quienes tienen la función de amplificadores, como el tubo en un instrumento viento; y en la lengua, quien junto con el paladar, dientes y labios articulan e impostan consonantes y vocales en el sonido amplificado. Aunque estos cuatro mecanismos funcionan independientemente, están sin embargo coordinados en el establecimiento de la técnica vocal y están hechos para interactuar unos con otros".

Según las definiciones anteriores, el canto es, en efecto, una actividad natural del ser humano; su correcto desarrollo depende de diferentes factores: físicos, personales y ambientales. A continuación, se presenta una breve descripción de la anatomía y fisiología del canto.

\section{Anatomía y fisiología del canto}

Para interpretar música con un instrumento, es necesario conocerlo, solamente así se buscará su mejor funcionamiento. De la misma forma, en el canto se necesita conocer los órganos implicados en la producción de sonidos; igual para hablar y para cantar.

\section{Sistema respiratorio}

Radinoff (2008) menciona en su libro The vocal instrument, que el sistema respiratorio es la fuente del sonido, y está compuesto por dos procesos: inhalación y exhalación. Al respecto, Maulèon-Stäheli (2015) lo denomina la "fuente de energía".

En la enseñanza de canto, una de las cosas que más se refuerzan es la buena respiración. Aquí se utilizan términos como appogio (Maulèon-Stäheli, 2015) y breath control/support (Radionoff, 2008) que en español significan "apoyo" y control/soporte de la respiración, respectivamente. Estos términos describen básicamente la entrada de aire correctamente o respiración abdominal (Naidich, Jackson, \& Segre, 1981), durante la cual el pulmón se oxigena desde la base hasta arriba, el diafragma desciende produciendo que el abdomen crezca hacia adelante y abajo generando una expansión de todo el cuerpo. Este tipo de respiración es el mismo que utilizan los bebés durante el nacimiento; una vez realizada la inspiración se hace una contracción energética de los músculos para la exhalación activa (Radionoff, 2008); los músculos abdominales actúan 
junto con el diafragma, promoviendo así el apoyo a la columna de aire necesario durante la emisión de los sonidos.

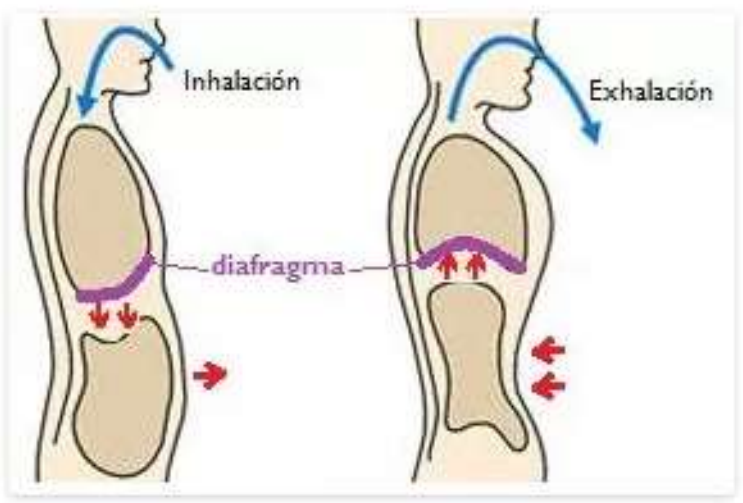

Ilustración 1

La ilustración 1 muestra gráficamente como debería ser la correcta respiración, tal como se explicó anteriormente.

\section{Sistema fonatorio}

Naidich, Jackson, y Segre (1981) mencionan que la faringe es el órgano productor del sonido. Sin embargo, éste sería inaudible si no contara con el refuerzo de las cavidades de resonancia; la técnica vocal debe enfocarse en "colocar las cuerdas vocales en posición fonatoria correcta"; además, dosificar el aire enviado a las mismas para producir una correcta afinación. En la adolescencia, este órgano sufre diferentes cambios que serán los responsables de la diferencia de voz masculina y femenina. En la ilustración 2 y 3 se observa la faringe y las cuerdas vocales.

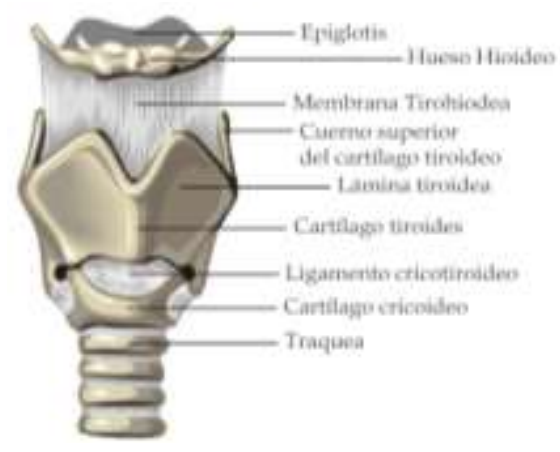

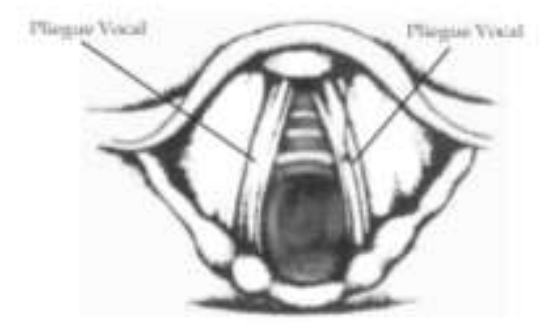

Ilustración 3

Resonadores 
Según Noriega (2002), el sistema resonador está compuesto por las estructuras que modelan la calidad y riqueza de la voz, la profundidad y pronunciación de las vocales consonantes; es decir, por la garganta, nariz, boca, paladar blando y senos craneales; según como se los acomode hacen cambiar los sonidos que se producen; por lo tanto, una persona que quiere cantar necesita conocer la forma como se debe ubicar cada sonido y la posición de las partes del cuerpo involucradas, para conseguir un buen sonido.

\section{Conceptos de canto coral}

Espinoza (citado por Cedeño, 2016) define el canto coral como: "la actividad musical orientada a la enseñanza de los aspectos básicos de la emisión vocal, como resonancia y respiración, en una práctica individual y colectiva”. Cedeño (2016), por su parte, concluye que "cantar en grupo desarrolla un modelo educativo de carácter inclusivo, donde se aprende y se desarrollan competencias musicales". Salvat (citado por Borda, 2018) afirma que el canto coral es "el agrupamiento de varias voces para el ejercicio de la interpretación musical por medio del canto" (p. 8).

Huamaní (citado por Matta, 2018) define el canto coral como "la acción realizada por un coro, con o sin acompañamiento instrumental, entendiendo por un coro a un grupo de cantantes que cantan juntos, bien al unísono o a varias voces, generalmente con más de un cantante por voz; se desarrolla tanto en la música popular (coros escolares, coros parroquiales, coros orquestales, otros) y la música académica escolástica (coro de cámara, coro sinfónico)" (p. 24).

\section{Voz adolescente}

$\mathrm{Al}$ considerar las edades de adolescentes de 10 a 19 años, se observan diferentes etapas de voces femeninas y masculinas, desde las voces blancas hasta la nueva voz de cada uno. Borda (2018) afirma que la muda de voz en mujeres es más rápida y menos notoria que en los hombres; en estos, el proceso dura de pocos días a muchos meses, variando por diversas razones.

Muda de voz femenina

Fase

Fase I (preadolescencia) sonido aflautado, propio de

voz infantil, y su sonoridad es similar a una soprano;

todavía la voz es similar a la

Descripción

La voz cantada posee aun un

\section{Características}


voz de un niño, pero con

menos volumen

$\begin{array}{llll}\text { Fase II-A } & \text { La voz cantada presenta una } & \checkmark & \text { Incomodidad al cantar } \\ \text { (adolescencia } & \text { tendencia hacia un sonido } & \checkmark & \text { Poca potencia sonora, dificultad } \\ \text { pre- } & \text { soplado, con exceso de aire } & & \text { para cantar fuerte. } \\ \text { menarquia) } & \text { (sobre todo en el agudo), } & \checkmark & \text { Voz muy aireada en el registro } \\ & \text { debido a la aparición de la } & & \text { de cabeza }\end{array}$

hendidura transitoria que impide cerrar

convenientemente la glotis.

Fase II-B Es la etapa más impredecible,

(adolescencia caótica, crítica y álgida de la post- muda de la voz femenina.

menarquia) Representa la cúspide de este proceso, la voz cantada atraviesa un momento crítico.

Fase III (voz El ámbito vocal se extiende juvenil de nuevo en esta etapa. femenina) Aquellas voces que poseen una mayor calidad presentan normalmente un amplio ámbito; también en el grave, se debe tener cuidado, porque se le puede confundir como contralto. Los registros de pecho y cabeza tienden a fusionarse.

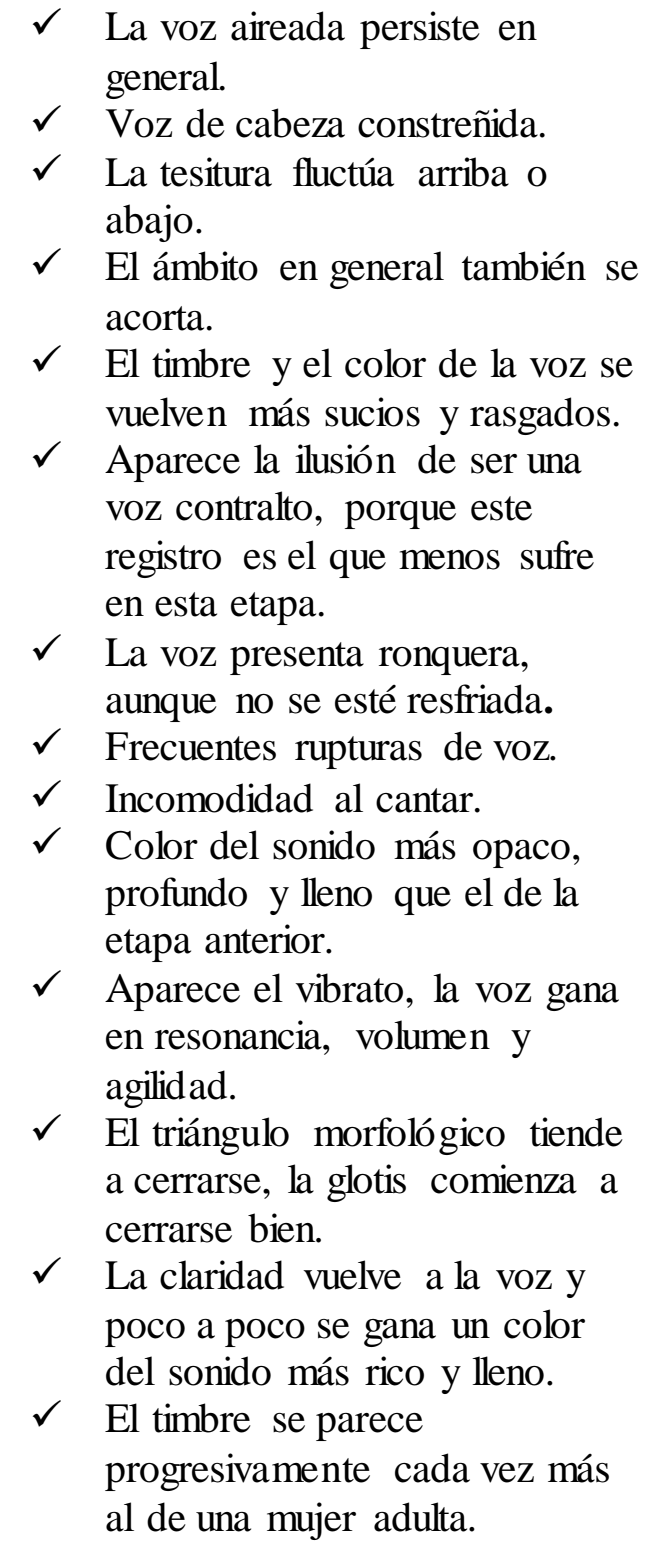

Tabla basada en Elorriaga (citado por Matta, 2018)

Muda de voz masculina

Elorriaga (2010) menciona que los cambios de las voces masculinas aparecen en la etapa de la pubertad. Empiezan con una repentina pérdida de registros agudos, sin incrementar los graves; luego el registro empieza a hacerse más grave, empezando por la 
voz hablada y luego la cantada. Además, la voz pierde brillantez, plenitud, intensidad, y redondez; se vuelve menos resonante y más débil, menos agil y más sensible a un esfuerzo vocal continuado. Durante la adolescencia la voz hablada se encuentra próxima a la voz cantada en la parte baja del registro; este se va a extender en la edad adulta. Según Cooksey (citado por Elorriaga, 2010), las etapas de la muda de voz masculina son las siguientes:
a) Voz blanca, infantil
b) Media voz 1
c) Media voz 2
d) Media voz $2^{\mathrm{a}}$
e) Nuevo barítono
f) Barítono en desarrollo o barítono joven hacia la voz adulta

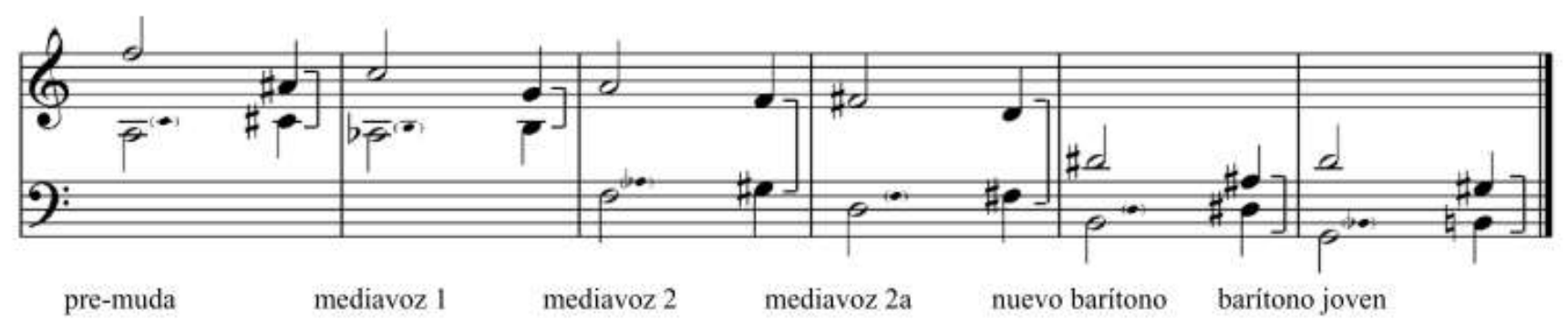

Illotración 4

Según la ilustración, las blancas muestran el ámbito vocal, mientras que las negras muestran la tesitura de la voz. Los cambios bruscos ocurren en esta etapa, mayorme nte en los varones. Borda (2018) aconseja no empezar clases formales de canto; por su parte, Elorriaga (2010) expone los principales problemas de emisión observados en esta etapa, los cuales son:

a) Voces masculinas: dificultades para cantar afinadamente por la desorientación auditiva que los chicos sufren cuando su registro desciende.

b) Voces femeninas: Aparición de una voz aireada en exceso con dificultades transitorias para cantar en el registro de cabeza, como consecuencia de una falta de madurez laríngea que impide cerrar convenientemente la glotis durante algún tiempo en la pubertad.

¿Significa entonces que en la edad adolescente no es posible cantar? Al respecto, Elorriaga (2010) afirma que los ejercicios debidamente realizados, en el caso de los varones, en tonos cercanos a su registro hablado, pueden afinar muy bien cuartas, quintas 
y octavas. En el caso de las mujeres, los cambios no son tan pronunciados como en el caso de los varones, ellas suelen tomar bien este cambio y, a pesar de algunas disfonías, ellas continúan normalmente esta actividad.

\section{Clasificación de las voces adolescentes}

\section{Vozfemenina}

La voz femenina se divide en: Soprano, la voz más aguda, Mezzosoprano, la voz intermedia entre las más aguda y la más grave; y Contralto, la voz más grave. Sin embargo, por los cambios previos en la voz, Huff-Gackle (citado por Elorriaga 2011) afirma que no se debería clasificar a las adolescentes en voz de Soprano o Alto, más bien están en un registro medio de Mezzosoprano; recomienda que las adolescentes realicen un mismo calentamiento vocal, las tesituras sean cómodas, se intercambien las partes vocales, a fin que tengan la oportunidad de cantarlas todas. Así, Rosabal (2009) explica que el error de hacer cantar a las adolescentes siempre en una misma tonalidad, generalmente contralto o notas medias-graves, hace que se acostumbren a cantar todo con voz de pecho, perdiendo la facilidad para cantar las notas agudas.

\section{Voz masculina}

Las voces masculinas son aproximadamente una 8va más grave que las voces femeninas; estas se clasifican en: Tenor, la voz más aguda; Barítono, la voz intermedia; y Bajo, la voz más grave. Así como en la voz femenina, no se puede clasificar a los adolescentes. Rosabal (2009) divide la clasificación de las voces masculinas adolescentes en dos etapas, de la siguiente manera:

\section{Primera etapa}

Esta etapa de cambio de voz es denominada cambiata (en italiano). Existen diferentes posturas de los registros que se deberían utilizar y las partes que se deberían cantar; se recomienda escoger un repertorio que tenga la voz cambiata; de lo contrario, realicen arreglos para que los adolescentes canten en un registro de La2 a su octava; además, vocalicen en su registro sin forzar su voz, para evitar que se generen vacíos entre la voz de pecho y la de cabeza, a la misma vez que se estimula y respeta el proceso natural de cambio.

\section{$\underline{\text { Segunda etapa }}$}


En esta etapa, los adolescentes cambiata pierden la facilidad de las notas agudas, lo que les impide continuar en este registro. Rosabal (2009) recomienda que, en esta etapa, se cante la parte del barítono o tenor, aunque no se consiga todas las notas, a fin de fortalecer más sus sonidos graves y su registro se irá ampliando hasta conseguirlo completamente. Collins (citado por Rosabal, 2009) recomienda que los adolescentes adopten una agrupación SSAB (Soprano, Soprano, Alto, Barítono), o la común SATB (Soprano, Alto, Tenor, Barítono) teniendo en cuenta el registro del cambiata.

\section{Estrategias pedagógicas}

La educación musical en cuanto al canto se realiza en un espacio, en el cual los adolescentes se expresen libremente; esto implica un trabajo constante (Elorriaga, 2010). En ese espacio, el adolescente puede relajarse y cantar diferentes melodías, permitiéndole gozar el deleite de cantar.

Por otro lado, a fin de desarrollar la voz y el canto coral, son necesarias algunas acciones; por ejemplo, la respiración es estudiada para realizarla correctamente, sin forzar las voces, evitando daño de los órganos vocales. Elorriaga (2011), en un estudio cuasiexperimental, investigó el impacto de la enseñanza de formación vocal en un grupo de adolescentes, cuyo grupo experimental demostró una mejora significativa en la actitud y auto-concepto para cantar, a pesar del cambio de voz, con una correcta pedagogía y formación.. Se demuestra el impacto de la pedagogía y la enseñanza para mejorar la emisión vocal y el concepto de los adolescentes sobre su propia voz.

Por su parte, el maestro debe crear ambientes agradables, mediante una enseñanza libre, llena de juegos y actividades guiadas, adecuada a la edad, estimulando el proceso de cambio de voz, y convirtiendo a los adolescentes en libres y cómodos para cantar, evitando la clase cargada. En este sentido, Elorriaga (2010) propone un programa vocal específico para educación secundaria que contenga las siguientes partes:

a) Explicación teórica de la muda de voz, que todos pueden encontrar su voz, pues cada voz es única y personal, desarrolla su propio ritmo; si se proponen, todos pueden aprender a cantar.

b) Iniciar el entrenamiento vocal basado en movimiento corporal y ejecución de ejercicios de coordinación psicomotriz.

c) Realizar actividades vocales para mejorar la capacidad auditiva, a través de un repertorio atractivo y adecuado a la edad.

d) Utilizar una estrategia práctica y efectiva para clasificar y modular las voces. 
e) Refuerzo a los estudiantes con problemas de coordinación auditiva y vocal, proveyéndoles ejercicios específicos para ayudarles.

Bennett (1986), por su parte, propone que, en la enseñanza vocal, cada estudiante:

a) Experimente la cualidad y rangos posibles de su voz.

b) Describa como siente su voz mientras canta, en qué parte del cuerpo la siente y cómo suena su voz y la de otros.

c) Coincida con la cualidad y rango de voz del profesor y otros estudiantes hablando o cantando.

d) Decida qué nombres definen mejor una producción vocal suya o de otro (por ejemplo "agudo", "medio" o "grave", "fuerte", "medio" o "suave")

e) Produzca un sonido particular según la especificación dada ("aguda", "suave", "tranquila", etc.).

f) Produzca una nota especifica dada por otra persona o instrumento.

En su planteamiento, Bennett (1986) estimula al docente a preparar un ambiente de canto al adolescente, en el cual éste se sienta lo más cómodo posible; una mala actitud o comentario negativo del profesor (o familiar del alumno) puede hacer que él/ella pierda el interés para desarrollar esta actividad coral.

\section{Preparación del instrumento}

A continuación, se describen los pasos para la preparación del instrumento vocal $\mathrm{y}$ algunos ejercicios sugeridos por diferentes autores:

\section{Relajación}

Para Naidich, Jackson, y Segre (1981, citados por Borda (2018), la relajación constituye una de las principales conquistas científicas de la impostación vocal moderna, se debe tener una relajación general y en especial de los músculos de la cara, lengua, mandîbula inferior, garganta y cuello, pues la relajación es la eliminación del trabajo muscular excesivo e innecesario. Según Borda (2018), hallar esta relajación no es un trabajo sencillo, se obtiene con la práctica; además resalta que es necesario realizar algunos ejercicios de relajación antes de empezar a cantar. En su tesis nombra seis ejercicios de relajación:

a) Elevar los hombros hacia arriba para dejarlos caer con todo el peso, mover hacia atrás y adelante haciendo círculos, este ejercicio se realizará de 2 a 3 minutos. 
b) Mover la cabeza en forma lenta hacia delante y hacia atrás, también a los dos costados y en forma circular, de 2 a 3 minutos.

c) Dar patadas al aire, dejando caer todo peso de las piernas, realizar este ejercicio de 1 a 2 minutos con cada pierna.

d) Hacer un puño con la mano, para realizar golpes suaves por todo el cuerpo, desde las piernas hasta la cabeza y la cara.

e) Entre los dedos pulgar e índice, tomar el mentón y movilizarlo hacia arriba, hacia abajo, también hacia los costados.

f) Estirar todo el cuerpo como si estuviéramos desperezándonos, acompañando los movimientos con una imagen natural de relajación.

\section{Respiración}

Respirar es un acto inconsciente y nadie necesita aprender a hacerlo; no obstante, para cantar es necesario tener conciencia de cómo se está respirando y cómo debería hacerse correctamente (respiración costo abdominal) para efectuar los cambios necesarios (Pineros, 2013, citado por Rivadeneira, 2015). Durante el proceso de respiración en el contexto del canto, la inspiración debe realizarse por la nariz, rápida, suave y silenciosamente. No demasiado profunda, la espiración debe realizarse por la boca y controlada por los músculos intercostales y abdominales; así como la relajación, ésta puede realizarse sentado, de pie o en marcha (Naidich, Jackson y Segre, 1981).

\section{Vocalización}

Para Borda (2018), este paso constituye un tipo de emisión basado únicamente en las vocales, aunque en un principio es necesario el uso de algunas consonantes, mientras se consigue el sonido esperado. En este proceso intervienen los órganos buco-laringofaríngeos, que actúan en conjunto coordinados para la producción de la voz (Balsebre et al., 2012). Durante la vocalización, Borda (2018) recomienda comenzar con la letra "I", luego la "E", "A", finalmente la "O" y la "U", utilizando las consonantes: "M", "N" o "L"; esto facilita la emisión de sonido y ayudan a colocar la voz en su lugar (Naidich, Jackson, y Segre, 1981).

\section{Beneficios del canto coral en adolescentes}

Los especialistas afirman los beneficios de la música para el organismo. Para Gardner (citado por Calderón-Ruíz y Pulido-Gordillo, 2015), la música es parte de una de las inteligencias múltiples, influye en el desarrollo emocional, espiritual y cultural, 
además que desarrolla el cerebro en sus dos hemisferios, por su carácter holístico. Por ejemplo, el proyecto Zero de la universidad de Harvard ofrece resultados positivos de la música; es ayuda y medio de aprendizaje de un idioma extranjero en inmigrantes recién llegados a Washington, o de disminución de tráfico de drogas en lugares expuestos a música clásica en vivo (Calderón-Ruíz y Pulido-Gordillo, 2015).

Por su parte, Barbosa (2014) sostiene que el canto coral mejora la identidad cultural, en el caso de la música autóctona desarrolla la empatía en el grupo; fomenta las habilidades sociales y es una extraordinaria herramienta para establecer una densa red de configuraciones socio-culturales mediante los eslabones de valoración de la propia individualidad, de la individualidad del otro $\mathrm{y}$ del respeto por las relaciones interpersonales; en un compromiso de solidaridad y cooperación. En opinión de Fucci Amato (citado por Barbosa, 2014), el canto es muy importante para el desarrollo de la personalidad; pues los adolescentes, durante un periodo de cambios y conocimiento de su voz, crean un auto-concepto e identidad y mediante las presentaciones en público refuerzan la confianza en sí mismos. A continuación, Cedeño (2017), por su parte, elabora una clasificación de los beneficios del canto coral, en las diferentes dimensiones: musical, pedagógico, cultural o actitud y personal:

\begin{tabular}{|c|c|c|c|}
\hline Desde lo musical & $\begin{array}{l}\text { Desd } \\
\text { pedagó }\end{array}$ & $\begin{array}{l}\text { Desde lo cultural o } \\
\text { actitud }\end{array}$ & Desde lo personal \\
\hline 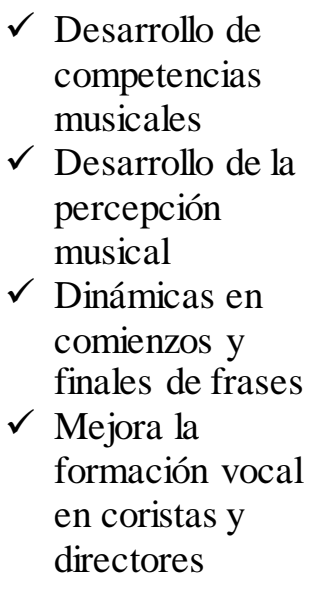 & $\begin{aligned} & \checkmark \text { Aprendizaje en } \\
& \text { grupo } \\
& \checkmark \text { El coro como } \\
& \text { instrumento de } \\
& \text { integración } \\
& \text { social } \\
& \checkmark \text { Se puede } \\
& \text { vincular con } \\
& \text { otras materias; } \\
& \text { por ejemplo, con } \\
& \text { educación física } \\
& \text { para el desarrollo } \\
& \text { de coreografías y } \\
& \text { de la motricidad. }\end{aligned}$ & $\begin{aligned} & \checkmark \text { A través del } \\
& \text { repertorio conoce } \\
& \text { historia y otras } \\
& \text { culturas. } \\
& \checkmark \text { Se desarrolla la } \\
& \text { interculturalidad. } \\
& \checkmark \text { Se reconoce y } \\
& \text { aprende otros } \\
& \text { idiomas } \\
& \checkmark \text { Construcción de la } \\
& \text { identidad vocal y de } \\
& \text { género } \\
& \checkmark \text { Aprende el valor de } \\
& \text { la música }\end{aligned}$ & $\begin{array}{ll}\checkmark & \text { Mejora la } \\
& \text { autoestima } \\
\checkmark & \text { Disminuye los } \\
& \text { niveles de } \\
\text { depresión y } & \text { estrés } \\
\checkmark & \text { Se fomenta la } \\
& \text { relajación } \\
\checkmark & \text { Se mejora la } \\
& \text { expresión facial } \\
\checkmark & \text { Desarrolla la } \\
\text { coordinación }\end{array}$ \\
\hline
\end{tabular}

En cuanto al canto coral para el bienestar psicológico, Stephen y Grenville (2010), describen los siguientes beneficios para los miembros de un coro: efecto positivo, atención enfocada, respiración profunda controlada, soporte social, estimulación cognitiva, compromiso regular. El esfuerzo de conseguir una buena música colectiva, 
proporciona hombres disciplinados y de noble carácter (Zuleta, 2004, citado por Rivadeneira 2015). Uno de los mayores beneficios es la socialización y trabajo en grupo; es una actividad que requiere de la cooperación de cada uno para conseguir un buen resultado.

\section{Conclusiones}

En este estudio, se suscriben las siguientes conclusiones: La voz adolescente está llena de cambios, los cuales duran algunos meses y son muy notorios; particularme nte, en los varones; poco perceptibles y cortos, en las señoritas. Durante este proceso, las voces pasan por diferentes cambios, son clasificadas de diferentes formas durante dicho proceso.

Al trabajar con adolescentes es necesario que el director y los mismos adolescentes conozcan su voz para clasificarla correctamente y hacer un buen uso de la misma; se debe escoger repertorio adecuado que contenga rangos adecuados para cada etapa, para fomentar el desarrollo de los diferentes tipos de voz. El canto trae grandes beneficios a los adolescentes, en los diversos aspectos: musical, académico, social, físico y de desarrollo personal.

\section{Referencias}

Barbosa, D. (2014). Beneficios del canto coral en adolescentes y su integración en el currículo de Música en la Educación Secundaria Obligatoria. Estudio sobre alumnado de un centro educativo de Cádiz (Tesis de maestria). Universidad internacional de $\mathrm{La}$ Rioja, . Recuperado de: https://seminariointegradorfamunam.files. wordpress.com/2015/03/canto-coral-enadolescentes.pdf $\% 5 \mathrm{Cn} \% 5 \mathrm{Cn}$

Bennett, P. (1986). A responsability to young voices. The Journal of the Texas Choral Directors Association, 73(1), 33-38. Retrieved from https://doi.org/10.2307/3400391

Borda Arana, M. E. (2018). Influencia del canto coral en la formación musical y personal de los(as) niños(as) del barrio de San Blas: 2014 - 2016 - Cusco. (Tesis de maestria). Universidad nacional de San Agustín de Arequipa, Arequipa, Perú.

Britannica, T. E. (2018). Singing. Retrieved October 5, 2019, from Encyclopadia Britannica website: https://www.britannica.com/art/singing

Calderón Ruíz, S. y Pulido Gordillo, M. (2015). El canto coral como estrategia 
pedagógica para el desarrollo de competencias ciudadanas que contribuyan al mejoramiento del clima de aula en los grados décimo y segundo de dos instituciones educativas distritales de Bogotá. (Tesis de maestria). Universidad de La Sabana, Cundinamarca, Colombia.

Cedeño Rómulo, L. C. (2016). El canto coral y el desarrollo de competencias musicales a través de un repertorio con elementos armónicos y melódicos del jazz en la comunidad de la fundación Renal del Ecuador Iñigo Alvarez de Toledo (Tesis de licenciatura). Universidad Católica de Santiago de Guayaquil, Guayaquil Ecuador. https://doi.org/10.1677/joe.0.1560509

Elorriaga, A. (2010). El coro de adolescentes en un instituto de educación secundaria: Un estudio de fonación. Revista Electronica Complutense de Investigacion Musical, 7, 1-20. Recuperado de: chromeextension://cbnaodkpfinfiipjblikofhlhlcickei/src/pdfviewer/web/viewer.html?file=h ttps://revistas.ucm.es/index.php/RECI/article/download/36939/35749

Elorriaga, A. J. (2011). Una propuesta de práctica de canto colectivo en la adolescencia : Un estudio de intervención en un IES. Revista Electrónica de la Lista Europea Electrónica de Música En La Educación, 28(28), 37-77. Recuperado de: http $/ /$ musica.rediris.es/leeme

Frühholz, S. y Belin, P. (Eds.). (2018). The Oxfordhandbook of voice perception. Oxford, Inglaterra: Oxford University Press.

Jaraba, M. Á. (1989). Teoría y práctica del canto coral. Madrid, España: Editorial Alpuerto.

Matta Alza, K. R. (2018). Aplicación del Taller de canto coral para el desarrollo de las habilidades sociales en estudiantes de IV año de Educación Secundaria de la IEP. "San Antonio Marianistas" - Bellavista - Callao (Tesis de maestría). Universidad César Vallejo. Lima, Perú. Recuperado de: chromeextension://cbnaodkpfinfiipjblikofhlhlcickei/src/pdfviewer/web/viewer.html?file=h ttp://repositorio.ucv.edu.pe/bitstream/handle/UCV/25338/Matta_AKR.pdf?sequenc $\mathrm{e}=1$ \&isAllowed $=\mathrm{y}$

Maulèon-Stäheli, C. (2015). Arte y ciencia. Hacer y pensar la pedagogía vocal. Revista de investigaciones en técnica vocal, 1, 78-87. Recuperado de: https://re vistas.unlp.edu.ar/RITeV/article/view/2061

Noriega Torres, E. (2002). La técnica vocal Hablada \& cantada. Miami, Estados Unidos: Caribe. 
Rivadeneira, F., X. (2015). Cancionero para coros infantiles de 8 a 12 años en base a ritmos ecuatorianos (Tesis de licenciatura). Pontificia Universidad Católica del Ecuador, Quito, Ecuador. Recuperado de: http//repositorio.puce.edu.ec/bitstream/handle/22000/9822/Cancionero infantil en base a ritmos ecuatorianos.pdf?sequence $=1$

Rosabal, G. (2009). Algunas perspectivas para el manejo de la voz adolescente en el ensamble coral. Eufonía didáctica de la música, (45), 50-57.

Stephen, C., \& Grenville, H. (2010). The significance of choral singing for sustaining psychological wellbeing: findings from a survey of choristers in England, Australia and Germany. Music Performance Research, 3(1), 79-96. 